\title{
Whose right to genetic knowledge?
}

A new section in this issue of Nature highlights the growing demands of insurers for access to genetic information, the diversity of legislative responses, and the many scientific uncertainties. The history of genetics suggests a controversial way forward.

FEW implications of modern genetics generate as much heated debate as its consequences for medical and life insurance. The central issue is straightforward: information about an individual's genetic profile can provide important clues about his or her future state of health, and therefore has a direct bearing on actuarial calculations about that individual's health and life expectancy. But, as a series of articles in this week's issue demonstrates - serving also to inaugurate Nature's new and occasional 'Briefing' section (pages 389-392) - how the implications should be tackled is less clear, and is the source of widespread disagreement. On the one hand, the insurance industry claims that access to genetic information is essential for the calculation of equitable premiums; on the other, the industry's critics warn of the undesirable effects that unregulated access could create.

The familial inheritance of certain disorders is already taken into account by insurance companies in determining whether to offer coverage to an individual, and, if so, on what terms. Meanwhile, gene-mapping and sequencing techniques, as well as the growing detailed knowledge of the relationship between genetic mutations and human disease, suggest that a rapid expansion is imminent in our ability to predict individual illness and mortality. Some late-onset illnesses (Huntington's disease is a well known example) can already be predicted with certainty from genetic mutations, whereas in some other diseases genetic information will at best deliver only an uncertain assessment of predisposition. But at both extremes of (un)certainty, such information is potentially equivalent in kind to other information already taken into account by insurers.

Nevertheless, public caution is widespread - partly because of lack of trust in insurers. The spread of HIV and AIDS has raised in an acute form the question of a company's right of access to the results of screening tests and how it should react to these results. And individuals already refuse to be screened for illnesses in case a positive result makes it impossible for them to obtain insurance at all. Such difficulties will increase rapidly as screening technologies develop.

It is easy, as some in the insurance industry are quick to point out, to exaggerate both our current abilities and the speed with which these are likely to grow. Much remains to be done in understanding not only how different mutations in individual genes may lead to a particular disease, but also how other diseases can result from an imbalance in the complex interaction of products of a number of different genes. Even more difficult to put into the quantitative terms required for actuarial calculations, is the impact, on both morbidity and mortality, of a combination of a genetic predisposition to a certain disease and the environmental factors that may either accelerate or delay its onset. For these reasons, perhaps, insurers are notably silent in response to enquiries about intended actuarial uses of genetic information.

In one sense, the interests of the industry and those it protects coincide: both seek to spread the burden of risk in an equitable fashion. But there is also an inherent conflict, in that both seek to maximize their own returns from the process. And there will inevitably be occasions, usually based on an imbalance of access to information, on which the efforts of the one to do so will reduce the returns to the other.

Take, for example, the question of 'adverse selection'. This is the situation in which an individual finds through a genetic test that he or she has a high chance of developing a life-threatening disease within the near future, and takes out a large insurance policy on standard terms - those based on an assumption of a normal life expectancy. In this case, the individual concerned is likely to emerge a clear winner, while either the insurance company, or other insurance holders if the company decides to pass on its costs as higher premiums, will be the loser. Faced with such possibilities - which would be entirely legal under present rules, which do not require applicants to disclose details of a disease they are not currently suffering from - it is not surprising that the industry's nervousness is increasing.

There are other reasons for the insurers to fret - not least, issues of commercial competitiveness. For example, a company which offers cut-rate insurance to those who have taken voluntary tests that reveal a relatively 'healthy' genome not only stands to make a considerable profit, but will also raise the proportion of those disease-susceptibility genes among competitors who do not discriminate. Yet at the same time, the industry will win few friends if it does introduce widespread discrimination, because the relatively low number of individuals required to pay a higher than average rate for life insurance could well rise substantially.

Equally justified are concerns on behalf of those applying for both medical and life insurance. The presence of questions about genetic testing on insurance application forms could deter some individuals from volunteering for screening tests that would otherwise be desirable. At the same time, there are ethical dilemmas for those who may prefer not to know the results of such tests (for example, the prospect of developing Alzheimer's disease).

As the years pass, the development of genetic tests and therapies will lead to a growth in confidence in the handling of such information. In the meantime, regulation is required. Here the successful experience of handling the safety aspects of genetic engineering provides a useful model; a short, sharp moratorium on use of genetic information by insurers combined with fierce public debate, followed by initial regulations that are deliberately over-cautious, and a subsequent period of liberalization as more becomes known of both the real and hypothetical dangers posed. Despite short-term problems and controversies, this is the best way in the longer term to develop public confidence in understanding both the tests and the way in which they are applied actuarially, and in the ability of regulators to curb abuse. But in the end, both insurer and policy-holder will have to share information equitably - there is no long-term alternative to consensual discrimination.

These issues loom large, but need also to be seen in the context of other far-reaching implications of current research, not least the future need (and desirability) for people and societies to adjust more generally to the predictabilities that will arise from genetic knowledge. Treatments of illness will also advance, but the scale of the necessary rethinking about our lives will make today's debates about insurance seem relatively trivial. 